\title{
论 文
}

\section{积云模式中上行地闪的参数化方案及起始有利 云内环境特征的探讨}

\author{
谭涌波*，陈超，周洁晨，周博文，张冬冬，郭秀峰 \\ 南京信息工程大学气象灾害教育部重点实验室, 气候与环境变化国际合作联合实验室, 气象灾害预报预警与评估协同创新中心, \\ 中国气象局气溶胶与云降水重点开放实验室，南京 210044 \\ *E-mail: ybtan@ustc.edu
}

收稿日期: 2016-01-18; 接受日期: 2016-03-18; 网络版发表日期: 2016-06-28

国家重点基础研究发展计划项目(编号: 2014CB441403)和国家自然科学基金项目(批准号: 41175003, 41475003)资助

\begin{abstract}
摘要上行地闪是一种始发于超高建筑物(高度至少在 $100 \mathrm{~m}$ 以上)顶端的大气放电现象, 目前对其的认知主要 通过地面观测, 而相应的理论模式研究较为缺乏. 本文在已有的双向先导随机模型的基础上, 创建上行地闪随 机放电参数化方案, 并耦合到雷暴云起、放电模式中, 进行了二维高分辨率上行地闪放电的模拟实验, 得到的上 行闪电与观测结果具有较好的一致性. 通过分析雷暴云电荷结构给出了常规地闪起始的有利云内环境特征, 并 分析了正、负上行地闪一些特征的异同, 结果表明: 模拟得到的上行正地闪多为诱导触发的上行地闪, 通常是三 极电荷结构下次正电荷区与地面之间的一种放电现象, 前次云闪过程对空间环境电场的影响为其起始提供了有 利条件, 整个放电过程延伸范围有限、分叉少、放电不充分; 上行负地闪多为偶极电荷结构中主负电荷区与地面 之间的放电过程，温度层结的高度低以及降水粒子的下沉使电荷区高度降低是其起始的根本原因，上行负地闪 发展旺盛, 分支较多; 诱导触发的上行地闪主要发生于雷暴成熟期, 而自行触发的上行地闪则更容易在雷暴消 散期起始.
\end{abstract}

关键词上行地闪, 数值模拟, 触发条件, 传播特性, 雷暴云模式

\section{1 引言}

当雷暴云过境的时候, 由于地面高大建筑、风塔 等会对周围大气电场的有着很强的畸变作用, 在一 些高大建筑物顶端以及风塔尖端会触发上行地闪(郄
秀书等, 2013). 近几年来, 由于社会在不断发展, 科 技在不断进步，全球范围内的高大建筑物以及风车 普遍增多, 以至于从高楼以及高塔上触发的上行地 闪备受关注(Miki等, 2005; Flache等, 2008; Wang等, 2008; Pichler等，2010；Lu等，2012；Warner，2012；

中文引用格式: 谭涌波, 陈超, 周洁晨, 周博文, 张冬冬, 郭秀峰. 2016. 积云模式中上行地闪的参数化方案及起始有利云内环境特征的探讨. 中国科学: 地 球科学, 46: 986-999, doi: 10.1360/N072015-00374

英文引用格式: Tan Y B, Chen C, Zhou J C, Zhou B W, Zhang D D, Guo X F. 2016. A parameterization scheme for upward lightning in the cloud model and a discussion of the initial favorable environmental characteristics in the cloud. Science China Earth Sciences, 59: 1440-1453, doi: $10.1007 / \mathrm{s} 11430-016-5309-5$ 
Zhou等, 2012a). McEachron(1939)最早观测到发生在 纽约帝国大厦的上行地闪. 在此之后, 在世界上越来 越多地区和国家都开展了上行地闪观测研究, 例如 以冬季雷暴为主的亚洲国家 (Takagi等, 2006; Wang 等, 2008; Lu等, 2009)、囊括所有季节雷暴的欧洲国家 (Miki等, 2005; Flache等, 2008; Diendorfer等, 2009; Romero等, 2011; Zhou等, 2012a) 以及以夏季雷暴为 主的美国(Mazur等, 2011; Warner, 2012; Warner等, 2012a, 2012b).

目前主要是通过地面电磁场观测以及高速摄影 等手段对上行地闪进行研究, 得到了关于上行地闪 的一些结论, 例如上行地闪的极性以及触发机制等. 一般而言, 当向上传播的单向先导为正先导时, 电流 方向向上, 定义其为上行负地闪, 反之则为上行正地 闪. 当先导通道内电流方向发生变化时上行地闪为 双极性上行地闪(Berger等, 1967, 1969; Rakov等, 2003; Diendorfer等, 2009). Diendorfer等(2011)统计了 2000 到2009年之间发生在奥地利 Gaisberg塔上的 651 次上行地闪, 其中 $93 \%$ 是负极性上行地闪, $4 \%$ 为正极 性上行地闪, $3 \%$ 为双极性上行地闪. 大量的观测结果 显示, 在冬季雷暴中负极性上行地闪在自然界中的 上行地闪中占主导地位(Eriksson等, 1978; Chang等, 1985; Manhardt等, 2012; Warner等, 2012c; Guimarães 等, 2014; Jiang等, 2014a, 2014b).

上行地闪究竟是如何从高大建筑物以及高塔等 尖端上触发的? 云内的电环境特征到底如何为上行 地闪的起始提供有力条件? Berger(1967)第一次提出 了上行地闪是否会被其他闪电触发的问题, Berger等 (1969) 认为云内放电产生一个迅速电场变化引起上 行地闪, 而不是缓慢的云内电荷积累过程. 可是, Orville和Berger(1973)认为上行地闪由强静电场触发, 而之前没有发现有任何闪电活动. 而Wang等(2008) 通过观测结果指出了上述两种情况都可以触发上行 地闪, 并指出上行地闪触发机制主要有两种, 一种是 诱导触发的上行地闪, 是由附近放电活动产生的强 大电场所激发的, 主要在强对流雷暴天气条件下更 容易产生; 一种是自行触发的上行地闪, 是由云内电 荷缓慢积累产生强大电场而引起的, 主要在非强对 流雷暴天气条件下容易产生. Wang和Takagi(2012)指 出诱导触发的上行地闪一般发生在放电中心高且活 跃的雷暴过程中, 而自行触发发生在闪电活动较少
的时候. Zhou等(2012b) 指出少数由其他闪电触发的 上行地闪大多数都发生在夏季的强对流雷暴天气 (4 8月), 这可能与夏季雷暴云有很高的电荷中心有 关; 大多数上行地闪是自行触发的上行地闪, 其中大 部分都发生在冬季非强对流雷暴天气(9月 次年3月), 并且认为导致自触发上行地闪概率高的原因与冬季 雷暴云电荷结构和建筑物相对周围地形的高度有 关; Warner等(2012c，2014)统计了2004 2010年发生 在Rapid City的上行地闪, 多数都是由其他闪电触发 的. 另外, 在 2011 年 2 月一次暴风雪天气环境下他们 观测到了有高达 $93 \%$ 的闪电为自行触发的上行地闪, 并分析暴风雪天气条件下的猛烈强风和较低的雷暴 云放电中心是自行触发的上行地闪发生概率较高的 原因. Carey等(2005)和Liu等(2013)都认为在雷暴云低 的电荷中心环境下, 云内闪电发生少, 云中电荷可以 进行缓慢而充分的充电, 致使容易产生上行地闪. 尤 其在雷暴云消亡阶段的时候, 闪电活动少, 更可以缓 慢增加底部电场强度. Jiang等(2014a)通过查看多普 勒雷达剖面图, 发现当上行负地闪电触发的时候, 雷 暴云底部电荷区很低在2 3 $\mathrm{km}$ 左右, 并且带有负电 荷. 由此可见, 上行地闪的触发类型与季节有很大的 关系: 冬季的时候自行触发的上行地闪比较多, 这可 能与冬季雷暴云电荷结构中心低有关; 夏季的时候 由其他闪电活动触发的上行地闪较多, 这可能与夏 季强对流雷暴云电荷结构中心高有一定的联系. 总 之, 目前关于上行地闪的研究主要是针对上行地闪 触发时的电环境特征进行探讨, 通过观测也累计了 一定的有意义的结论, 不过对上行地闪触发时云内 电环境特征也只是一些推测, 具体情况还不是很清 楚, 上行地闪触发的原因依然还在探讨之中, 以及其 他闪电活动对上行地闪触发的影响仍需进一步研究. 并且由于云体的遮挡, 上行地闪入云后的各种特 征以及对云内电荷的影响目前也还缺乏有效地研究 手段.

由于受上行地闪触发的随机性以及观测手段的 限制, 目前要获取上行地闪始发、传播时空中、地面 全部电参数难度较大. 需要建立相应的理论模型进 行讨论, 然而大部分都是探讨下行地闪连接过程中 的上行先导, 有了一定的基础, 如 Becerra和 Cooray (2006a，2006b)建立了上行先导传播的一种物 理模式, 模拟从各种各样的接地建筑产生的上行先 
导; 贺恒金等(2008)考虑先导发展概率与局部电场的 随机关系, 模拟了闪电先导与传输线的相互作用; 任 晓毓等(2010)结合随机放电理论建立了一个闪电先 导的二维模型, 分析了下行先导与建筑之间的相互 作用和连接过程. 虽然上行先导的模式工作已有了 初步的发展, 但是上行地闪模式开发工作进展缓慢, 目前关于上行地闪的模式研究工作还少见报道, 并 且由于分辨率的限制, 在一次完整雷暴过程中产生 上行地闪的模式少有研究, 并且其他闪电活动影响 上行地闪触发的模式更需要开拓. 基于此, 本文在已 有的双向先导随机模型(Mansell等, 2002; 谭涌波等, 2006)的基础上，创建能够自行触发的上行地闪随机 放电参数化方案, 并耦合到雷暴云起、放电模式中, 再通过大量敏感性实验, 模拟上行地闪的触发以及 传播入云过程, 探讨不同雷暴云电荷结构以及其他 闪电活动对上行地闪触发以及传播的有利条件, 并 且比较产生上行地闪与下行地闪时的云中背景条件 的差异.

\section{2 模拟方法}

本文首先在已有的双向先导随机模型 (Mansell 等, 2002; 谭涌波等, 2006)的基础上, 建立上行地闪 随机参数化方案, 并将其耦合到已有的二维雷暴云 起、放电模式中(谭涌波等, 2007, 2012; Tan等, 2014a). 其中起电机制采用了改进的非感应起电机制(Tan等, 2014a; Shi等, 2015) 以及感应起电机制 (Ziegler等, 1991), 而云闪以及地闪参数化方案具体见参考文献 (Tan等, 2006; Tao等, 2009), 这里不再累述. 需强调 的是, 之所以采用二维模式, 是因其可在高分辨率下 进行闪电放电的模拟. 在计算微物理-动力以及起电 过程时采用粗分辨率 (250m), 而一旦空间电场强度 达到了要求, 则嵌套到高分辨率 (12.5m)网格点上进 行放电模拟(Tan等, 2006; 谭涌波等, 2006, 2007). 本 文着重介绍新建立的上行地闪随机参数化方案.

\section{1 上行地闪放电参数化方案}

与云闪、地闪类似, 上行地闪参数化方案需考虑 上行地闪的启动、传播、终止以及通道内部感应电荷 的计算等问题. 虽然上行地闪起始于地面, 不同于始 发于云内的云闪与地闪, 但其也具备先导放电的特
征, 因此上行地闪的各个主要参数以及处理方法均 参考了随机放电参数化方案, 主要区别在于上行地 闪的启动以及先导的单向传播等问题上.

\subsection{1 上行地闪的启动}

上行地闪主要发生在超高建筑物 (高度至少在 $100 \mathrm{~m}$ 以上)之上(郄秀书等，2013; Rakov等，2003; Jiang等, 2014a), 建筑物对环境电场的畸变加强使其 超过上行地闪起始阈值 $E_{\mathrm{be}}$ 是能够触发上行地闪的根 本(郭秀峰等, 2013; Guimarães等, 2014). 目前关于上 行地闪的模式还很少见, 其起始阈值也很难测量并 且也没有准确的值, 大多数都是对上行先导触发以 及传播的研究. Petrov和Waters(1995)曾提出电场畸变 临界范围(CRFI) 的概念, 认为棒形物体前方的流注 长度达到 $0.7 \mathrm{~m}$, 且该区域范围内的电场达到 $500 \mathrm{kV} / \mathrm{m}$ 时, 上行先导就能够触发. 基于此, 任晓毓等(2010) 在模拟下行地闪连接过程中的上行连接先导时也采 用了 $500 \mathrm{kV} / \mathrm{m}$ 作为起始阈值, 考虑到上行地闪与上行 先导触发的物理机制是一样的, 均为始发于建筑物 尖端, 利用建筑物尖端对环境电场的畸变击穿空气. 因此本文设定上行地闪的起始阈值 $E_{\mathrm{be}}$ 同样为 500 $\mathrm{kV} / \mathrm{m}$.

要想在积云起、放电模式中模拟出上行地闪, 关 键问题是如何在地面产生 $500 \mathrm{kV} / \mathrm{m}$ 的强电场. 一般而 言, 由于在雷暴条件下近地面附近会产生很厚的电 荷屏蔽层(Qie等, 1994), 所以在上行地闪产生之前的 地面电场一般不会超过 $10 \mathrm{kV} / \mathrm{m}$, 根本达不到上行地 闪起始阈值. 因此, 需在下垫面增加一个建筑物, 而 已有的研究发现, 建筑物的高度以及模拟域的空间 分辨率均是影响建筑物尖端畸变计算的主要因素(郭 秀峰等, 2012, 2013; Tan等, 2014b), 其中建筑物尖端 电场畸变系数与分辨率呈指数增加的关系. 虽然目 前的放电模块采用了 $12.5 \mathrm{~m}$ 的高分辨率, 但与真实的 连续空间差别仍然巨大. 为解决这个问题, 本文虚拟 在地面设计一个 $300 \mathrm{~m}$ 高的建筑物, 不考虑其宽度, 并通过已有的电场畸变系数与建筑物所在的模拟域 采用的空间分辨率的计算公式(郭秀峰等, 2012), 直 接给出建筑物尖端的电场强度, 来判断是否达到闪 电启动条件, 而不模拟建筑物对环境电位场的畸变 过程.

$$
\lambda=1202.89 \exp (-h / 0.08766)+112.15 .
$$


式(1)为建筑物在 $500 \mathrm{~m}$ 时的电场畸变系数 $\lambda$, 与 空间分辨率 $h$ 的计算公式. 因此在分辨率 $h$ 为 $12.5 \mathrm{~m}$ 的 情况下, $500 \mathrm{~m}$ 处的畸变系数为: $\lambda(12.5,500)=$ $1202.89 \exp (-12.5 / 0.08766)+112.15 \approx 112.15$. 而本文 的建筑物高度为 $300 \mathrm{~m}$, 并且畸变系数与建筑物的高 度成正比(郭秀峰等, 2013), 所以在 $12.5 \mathrm{~m}$ 分辨率下, 在高为 $300 \mathrm{~m}$ 的建筑物顶部畸变系数为: $\lambda(12.5,500) /$ $\lambda(12.5,300)=500 / 300 ; \lambda(12.5,300) \approx 67$. 通过触发间值 $E_{0}=500 \mathrm{kV} / \mathrm{m}$ 以及畸变系数 $\lambda(12.5,300) \approx 67$, 得到了 所需环境电场 $\mathrm{E}$ 为: $E=E_{0} / \lambda(12.5,300)=500 \mathrm{kV} / \mathrm{m} / 67 \approx$ $8 \mathrm{kV} / \mathrm{m}$.

为了订正计算过程的误差以及其他因素的影响, 本文将 $E$ 近似取为 $8 \mathrm{kV} / \mathrm{m}$. 也就是说, 当超高建筑物 的高度为 $300 \mathrm{~m}$, 其顶端的环境电场达到 $8 \mathrm{kV} / \mathrm{m}$ 时, 此 处存在的建筑物对环境电场产生畸变, 通过环境电 场乘以畸变系数 $\lambda(12.5,300)$, 得到畸变后的电场为 $500 \mathrm{kV} / \mathrm{m}$, 达到了触发阈值.

本文着重讨论上行地闪的模拟, 而雷暴云在整 个发展过程中会进行移动, 而一般而言雷暴云电荷 结构的正下方为地面环境电场最强的地方. 为使上 行地闪更容易触发, 本文设置的建筑物并不固定, 可 认为其随着雷暴云的移动而移动, 根据前面的模式 设置, 只要雷暴云下高度为 $300 \mathrm{~m}$ 处的环境电场达到 上行地闪的起始阈值, 则认为建筑物位于此, 上行地 闪产生; 如果存在多个超过触发阈值的点, 则认为建 筑物在环境电场最大的地方.

值得一提的是, 一些研究工作发现上行正、负先 导的起始、传播条件存在差异(Alessandro等, 2001; Solomon等, 2001), 但是针对这种差异到底有多大, 正负先导各自的阈值各为多少的问题, 尚未有文献 给出具体的解释. 目前几乎所有与闪电相关的模拟 工作中, 正负先导的阈值取值均相同 (Heldson等, 1992; Mansell等, 2002; Becerra等, 2006a, 2006b; 贺 恒金等, 2008). 鉴于此, 本文也暂未考虑正负先导的 差异性.

\subsection{2 上行先导的传播}

上行地闪具有与云闪、地闪相似的分叉结构(Tan 等, 2006; 谭涌波等, 2006; Tao等, 2009), 因此在描 述上行地闪传播时, 仍采用随机放电参数化方案 (Mansell等, 2002; Tan等, 2006; 谭涌波等, 2007), 通
过判断通道与环境之间的电位差是否超过传播阈值 $\left(E_{\mathrm{crit}}\right)$ 来判断通道的延伸. 由于上行地闪一旦形成, 其先导顶端温度极高, 使尖端附近的空气呈高度电 离状态, 这时要击穿空气使先导继续传播的电场临 界阈值会比触发阈值小很多, 并且上行地闪与云闪、 地闪一样, 均为空气击穿过程, 因此其 $E_{\text {crit }}$ 同样设置 为 $150 \mathrm{kV} / \mathrm{m}$ ( Tan等, 2006; 谭涌波等, 2006, 2007). 如 果有多个地方同时满足传播条件, 则通过概率函数 随机选取一个作为通道的后继扩展点(Tan等, 2006; 谭涌波等, 2007).

目前, 对于始发于云内的闪电均采用双向先导 理论, 正负先导同时产生、延伸. 正先导与环境之间 的电位差大于 $150 \mathrm{kV} / \mathrm{m}$, 即正先导沿着电位减少的地 方传播; 负先导则与之相反, 沿着电位增加的地方传 播, 而与环境之间的电位差小于 $-150 \mathrm{kV} / \mathrm{m}$, 这里的 正负代表方向而不是大小(Tan等, 2006; 谭涌波等, 2007). 上行地闪始发于建筑物顶端, 模式中认为建 筑物为良导体并与地球连接, 因此上行地闪为单向 传播的先导过程. 考虑上述多重因素, 上行地闪为单 向传播, 其传播阈值选取为 $150 \mathrm{kV} / \mathrm{m}$ 的绝对值, 即通 道与环境之间的电位差可以大于 $150 \mathrm{kV} / \mathrm{m}$, 也可小于 $-150 \mathrm{kV} / \mathrm{m}$, 当为正值时, 先导极性为正, 反之则极性 为负. 另外, 与 $T a n$ 等 (2006) 类似, 认为先导通道为有 电阻的导体, 通道内部压降 $E_{\mathrm{int}}$ 为 $500 \mathrm{~V} / \mathrm{m}$, 如果为正 先导则通道内部电位随着先导的延伸, 逐渐降低, 而 负先导的内部电位则逐步升高(式2).

$$
\phi(m)=\Phi_{r e f}-s \sum_{i=1}^{m} E_{\mathrm{int}} d_{i},
$$

式(2)中 $s$ 为通道极性, $\Phi_{\mathrm{ref}}$ 为起始点参考电位, 由于上 行地闪起始于建筑物顶端, 因此其为 $0 \mathrm{~V}$. 考虑通道 对环境电位分布的影响, 当每完成一步新的通道扩 展后, 需调整空间电位分布. 将建筑物和扩展后的闪 电通道当作固定边界条件(第一类边界条件), 通过超 松驰迭代技术解泊松方程、重新计算得到新的空间电 位分布(Tan等, 2006; 谭涌波等, 2007).

需特别指出的是, 由于在上行先导起始时, 通过 式(1)将环境电场换算到了空间分辨率为 $1 \mathrm{~m}$ 时的情 况, 实际放电模块的空间分辨率 $12.5 \mathrm{~m}$, 而由于空间 分辨率对畸变电场的影响(郭秀峰等, 2012), 上行地 闪起始后, 如果利用超松弛迭代技术解泊松方程的 方法计算电位，其尖端与环境之间的电位差达不到 
放电的要求. 为了让上行先导能够顺利发展, 模式中 人为设置一个垂直向上传播的先导. 也就是说先导 的起始阶段为固定的先导, 其后在根据先导与环境 之间的电位差判断先导的发展延伸. 具体方法如下: 首先根据周围的电位极性, 判断假定的上行先导的 极性, 然后判断尖端电位与环境电位之间的电场强 度绝对值是否大于传播阈值, 如果满足则上行地闪 自由发展; 反之, 则人为规定通道点的正上方的点为 下一次的通道点, 然后重复之前的步骤, 直至满足传 播条件为止; 当人为垂直发展的通道点达 100 个 $(1250 \mathrm{~m})$ 时, 仍未达到传播要求, 则禁止此次上行地 闪触发.

\subsection{3 先导的终止以及感应电荷计算}

在模式中, 当所有的上行地闪先导通道点与其 周围环境点之间电场强度的绝对值都小于传播阈值 $E_{\text {crit }}$ 时, 或是上行地闪的通道达到了边界(非地面)时, 上行地闪过程结束.

处在一定空间电位分布下的闪电通道将产生感应 电荷. 在计算通道内感应电荷的时候, 将闪电通道近似 地看为一个导体. 根据高斯定理, 由于导体内部电位为 常数, 则得到导体表面的面电荷密度的计算式(3):

$$
\varepsilon \frac{\partial \varphi}{\partial n}=-\sigma,
$$

$\varepsilon$ 其中为空气的介电常数, $n$ 为导体表面的法线方向, $\sigma$ 为导体表面的面电荷密度.

\subsection{4 一次上行地闪的定义}

严格来说, 一次上行地闪到底如何定义目前尚 无定论, 不过主要是根据传播距离来判断的. 就模式 而言将传播距离在 $3 \mathrm{~km}$ 以上的闪电, 定义为一次上行 地闪. 而模拟得到的一些传播距离小于 $3 \mathrm{~km}$ 的放电行 为, 本文暂不将其视为一次上行地闪, 当然也有一些 观测发现上行地闪是没有入云的(Warner等, 2012a).

\section{2 个例及起始参数选取}

本文选择两个雷暴过程的探空曲线作为模拟的 起始场: 一个是1999年7月至8月期间, 在新墨西哥州 Langmuir实验室所在地进行了雷暴云内电过程的综 合研究 (Studies of Electrical Evolution in Thunderstorms, 简称SEET). 实验场地海拔高度大约 $3 \mathrm{~km}$, 位
于山坡上. 本文选用其中7月31日的雷暴个例进行了 数值模拟; 另一个为北京 1992 年 8 月 3 日一次中尺度 对流天气系统通过北京地区时的暴雨过程(简称 BJ83). 两个雷暴个例一个是山地雷暴, 另一个为平 原雷暴, 分别代表了两种类别的探空曲线, 其电荷结 构离地面的高度具有明显差异, 而电荷结构与地面 之间的距离可能是产生上行地闪的一个主因, 这有 利于本文对不同类型上行地闪的模拟和研究.

本文构建的模拟域为 $76 \mathrm{~km} \times 20 \mathrm{~km}$ ，时间步长为 $2 \mathrm{~s}$, 云模式的空间分辨率为 $250 \mathrm{~m}$, 放电模块的空间 分辨率为 $12.5 \mathrm{~m}$. 模式的起始扰动为一个水平半径为 $5 \mathrm{~km}$, 垂直半径为 $1 \mathrm{~km}$ 的温湿泡扰动, 其中心位于模 式区域中心左侧高度为 $1 \mathrm{~km}$ 的格点上. 需说明的是, 本文主要讨论的是既定电荷背景下的上行地闪特征, 而不讨论雷暴云的演变过程, 云的动力-微物理框架 以及起电过程只是为了提供产生上行地闪的各种电 荷背景. 因此为了增加上行地闪的模拟个例, 本文在 探空曲线固定的前提下, 通过调整中心最大温度和 相对湿度扰动这个两个参数, 进行多次模拟实验.

\section{3 模拟结果}

本文通过调整位温以及相对湿度这 2 个参数, 进 行了 44次雷暴过程模拟, 共计产生了251次上行地闪. 需特别指出的是本文中模拟得到的上行地闪频次偏 多, 其主要原因是因为模式中建筑物所在位置视雷 暴云底部最大电场位置而定, 与真实情况存在差异, 其目的为了增加模拟得到的上行地闪样本, 使模拟 结果更具有代表性.

\section{1 正、负上行地闪的对比分析}

图1、图2分别展示了在两次不同个例所发生的上 行正地闪和上行负地闪, 依次分别命名为 $\mathrm{sp} 1 、 \mathrm{sp} 2$ 、 $\mathrm{sp} 3$ 和 sn 1、sn2、sn3, 并且每次上行地闪都发生在不 同的雷暴过程中. 模式中设置的一次雷暴过程为 $80 \mathrm{~min}$, 整个雷暴过程分为三个阶段：起始阶段 (0 30min)、成熟阶段 (30 60min)、消亡阶段 (60 80min). 表1给出了各次上行地闪的所发生的详 细情况，包括产生上行地闪的个例、发生的时间、在 雷暴过程的时间段、极性以及上行地闪触发之前最近 一次产生的闪电类型与时间. 

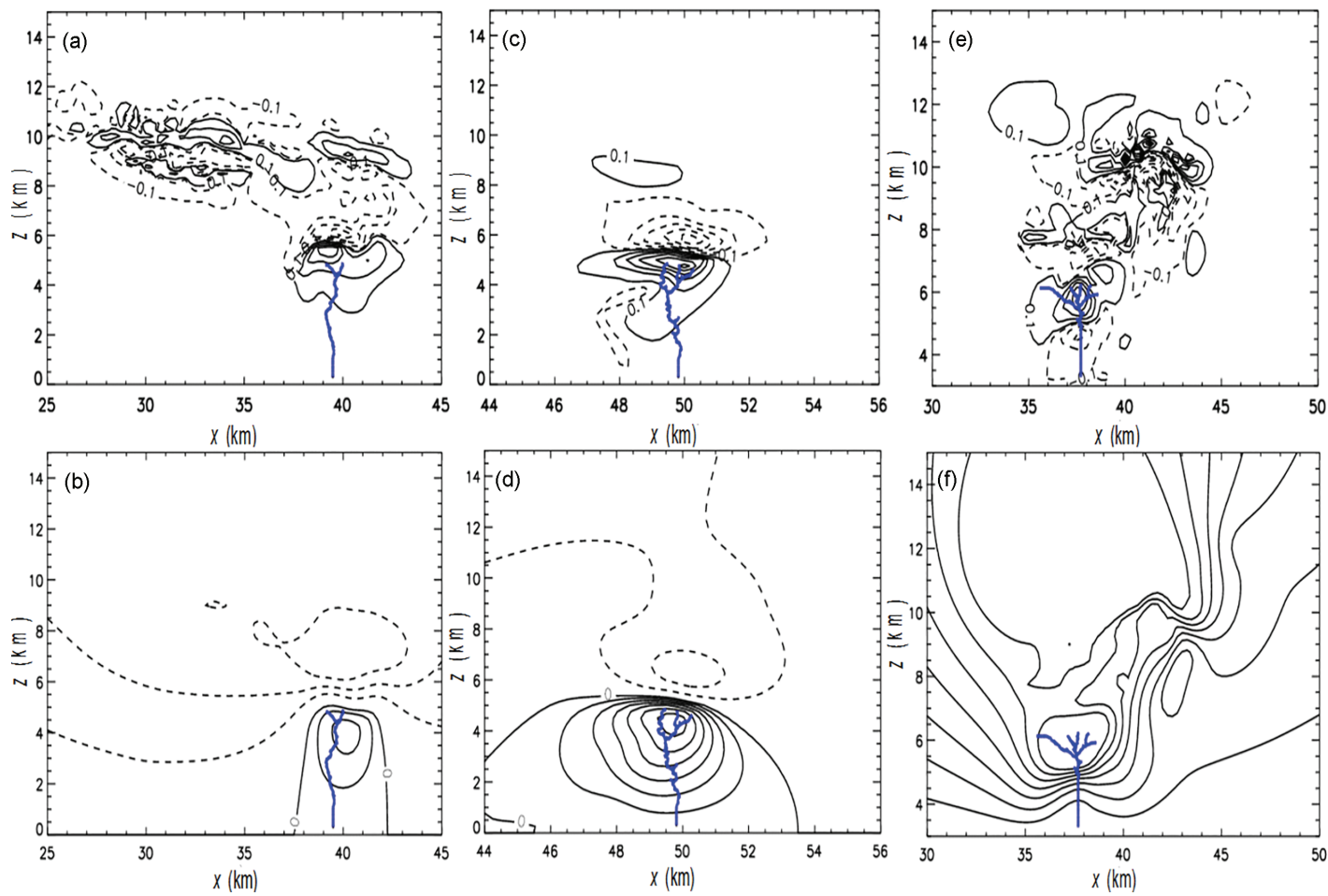

图 1 不同电荷结构下产生的上行正地闪电通道与空间电位以及空间电荷的分布图

蓝色实线表示上行正地闪电先导通道, 先导极性为负; (a)、(c)、(e)中实线和虚线分别代表正、负电荷密度等值线, 图中标定了 $\pm 0.1 \mathrm{nC} / \mathrm{m}^{3}$ 电

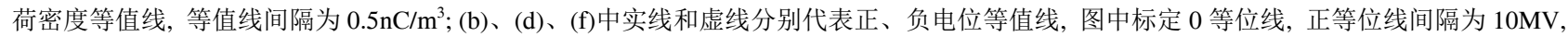
负等位线间隔为 $40 \mathrm{MV}$, 需要说明的是底边界也为 0 位势, 图中没画建筑物轮廓
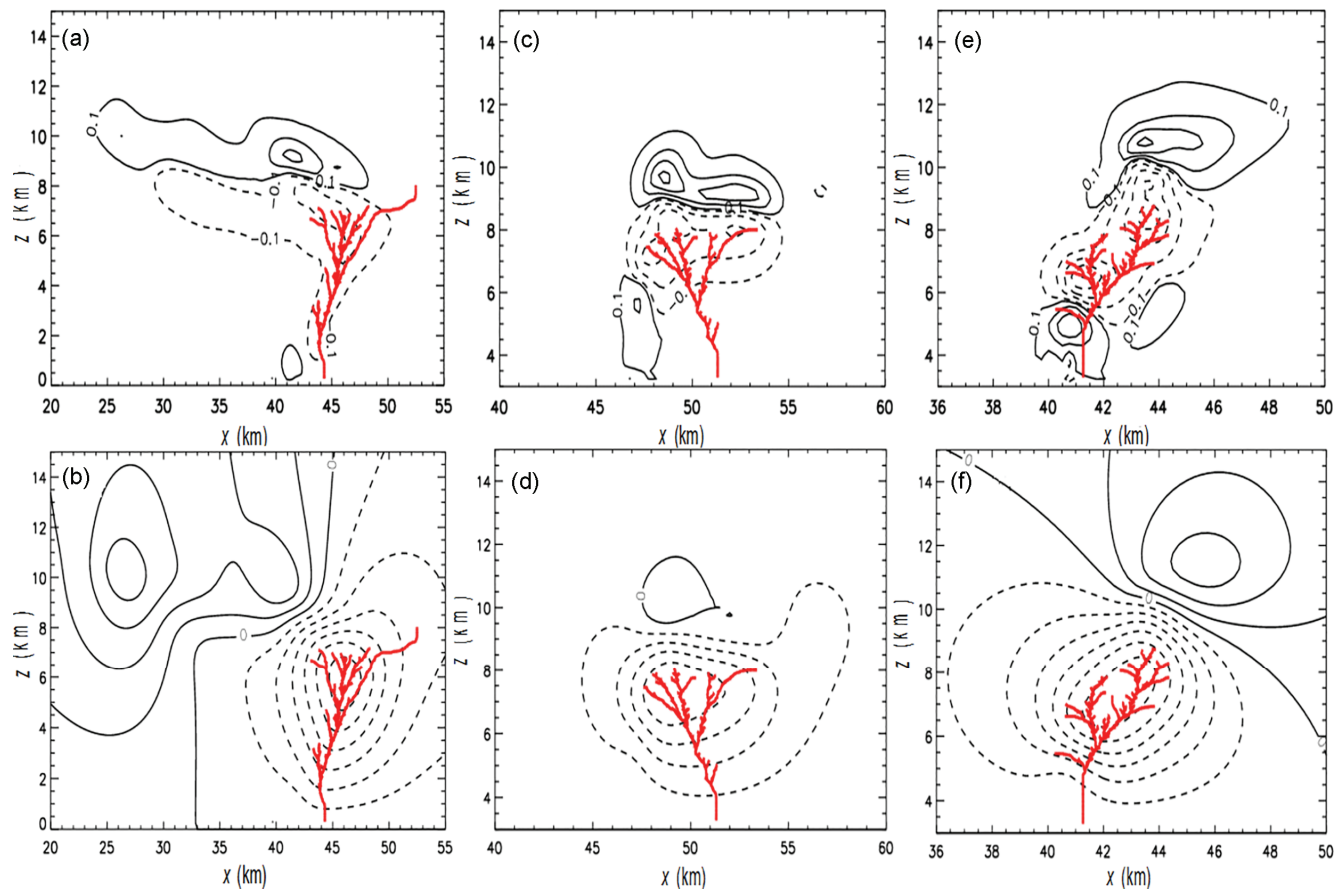

图 2 不同电荷结构下产生的上行负地闪电通道与空间电位以及空间电荷的分布图 正负等位线间隔为 $40 \mathrm{MV}$ 
从图1和图2中可以看出, 无论是上行正地闪还 是负地闪都起始于雷暴云底部电荷区的下方, 并且 产生的负(正)先导都是单向的、向着底部电荷高值中 心快速延伸，即底部的正、负位势阱中心传播. 在进 入位势阱中心之前, 正负上行地闪明显趋向于单支 垂直扩展，而分叉较少; 当传播至位势阱中心后，由 于通道与环境之间的电位差增大、各个方向的传播概 率增强，导致先导通道形成大量分支结构; 穿过位势 阱中心之后，在到达空中0位势等值线之前，上行正 (负)地闪迅速终止. 究其原因, 可能是因为位势阱会 吸引极性相反的先导向中心延伸，而阻碍其向外发 展(MacGorman等, 2001; Tan等, 2006). 已有的一些观 测结果表明在上行地闪触发点的几百米内先导都是 单支垂直发展的，当到云底下方附近时才会出现明 显的分支(Wang等，2008; Warner，2012; Warner等， 2012a, 2012c; Jiang等, 2014a, 2014b), 说明本文的模 拟得到的上行地闪特征具有一定的合理性. 另外, $\mathrm{sp} 3$ 以及 $\mathrm{sn} 3$ 这两个上行地闪个例, 均分别穿过了与先 导通道极性相同的底部电荷区, 达到了更高的与先 导极性相反的高密度电荷中心. 这是因为虽然底部 存在与先导通道极性相同的电荷区, 但是其上部的 异极性电荷区电荷量相对较大, 使得底部的位势阱 的极性与上行先导极性相反, 底部电荷区的存在并 不足以改变底部空间位势的极性, 而通道的延伸是 通过通道与环境之间的电位差决定的(Tan等, 2006, 2014a), 因此底部同极性电荷区的存在无法阻碍上行 先导的传播, 反而被其穿过. 与此同时, 虽然上行正 (负)地闪均始发于建筑物的顶端, 但通过图 1 、2以及 表1进行对比不难发现: 两种类型的闪电不管是从电 荷、电位背景, 传播的垂直、水平范围, 还是分形特
性上都具有较大差异.

\subsection{1 上行地闪的电位与电荷分布}

从图 1 和图 2 中可以看出上行正地闪 $\mathrm{sp} 1$ 和 $\mathrm{sp} 2$ 发 生的时候, 虽然在整个模拟域顶部全是负电位, 不过 底部会有一个强的正位势阱，而 $\mathrm{sp} 3$ 的整个模拟域均 为正电位，与此同时底部也存在一个正位势阱. 同样 地，上行负地闪 $\mathrm{sn} 1 、 \mathrm{sn} 2$ 以及 $\mathrm{sn} 3$ 发生的时候模拟域的 下方都会存在一个较强的负位势阱. 表2列出了6次 上行地闪发生之前模拟域中正、负位势阱的最大值 (上行正地闪)和最小值(上行负地闪), 显然负位势阱 中心最大值的绝对值比正位势阱要大很多, 产生上 行负地闪电背景条件更强. 总的来说, 上行地闪的触 发, 需要底部触发点附近要有强的位势阱, 并且位势 阱的极性与上行地闪的极性是相同的，正位势阱产 生上行正地闪，而负位势阱产生上行负地闪。

但通过对比上行地闪通道与空间电荷分布图, 不难发现其电荷结构却千差万别. 其中 $\mathrm{sp} 1$ 以及 $\mathrm{sp} 3$ 两 次上行正地闪触发时, 空中电荷结构比较特别, 上部 主负以及主正电荷区比较复杂 (见图1a、1e), 这是由 于分别在 18 个和 13 个时间步长内 (时间步长为 $2 \mathrm{~s}$ ) 触发 的一次云闪过程(见表1)在其对应的主正、负电荷区 中植入了异极性电荷(谭涌波等, 2007; Tao等, 2009), 显然这种前次云闪为后继上行地闪的触发提供了有 利条件，其与Wang等(2008)定义的诱导触发的上行 地闪比较相似; 而剩下的4次上行地闪至少在其之前 的7分钟内 (见表1)没有云内闪电发生，其空间电荷结 构为比较均匀的三极或反偶极结构并没有异极性电 荷植入空间电荷堆中，其应属于自行触发的上行地 闪(Wang等, 2008).

表 1 各次上行地闪的详细情况

\begin{tabular}{|c|c|c|c|c|c|c|}
\hline \multirow{2}{*}{ 名称 } & \multirow{2}{*}{ 个例 } & \multirow{2}{*}{ 时间 (min) } & \multirow{2}{*}{ 时间段 } & \multirow{2}{*}{ 极性 } & \multicolumn{2}{|c|}{ 最近一次闪电情况 } \\
\hline & & & & & 时间 & 类型 \\
\hline $\mathrm{sp1}$ & BJ83 & 47.00 & 成熟阶段 & 正 & 46.40 & 云闪 \\
\hline $\mathrm{sp} 2$ & BJ83 & 69.97 & 消亡阶段 & 正 & 62.90 & 负地闪 \\
\hline $\mathrm{sp} 3$ & SEET & 44.17 & 成熟阶段 & 正 & 43.73 & 云闪 \\
\hline $\operatorname{sn} 1$ & BJ83 & 61.23 & 消亡阶段 & 负 & 46.40 & 云闪 \\
\hline $\operatorname{sn} 2$ & SEET & 74.63 & 消亡阶段 & 负 & 64.37 & 云闪 \\
\hline $\operatorname{sn} 3$ & SEET & 55.73 & 成熟阶段 & 负 & 42.43 & 云闪 \\
\hline
\end{tabular}

表 2 生成各次上行地闪的位势阱中心电位最大值的绝对值

\begin{tabular}{cccccc}
\hline & sp1 & sp2 & sp3 & sn1 & sn2 \\
\hline 电位最大值的绝对值 (MV) & 24.4 & 65.3 & 109 & - & - \\
电位最小值的绝对值 (MV) & - & - & - & 264 & 199 \\
\hline
\end{tabular}


其中诱导触发的上行地闪 $\mathrm{sp} 1$ 发生的背景电荷结 构为经典三极性电荷结构 (图 1a), 而 sp3 的背景电荷 结构可以看做四极电荷结构(图1e), 次正电荷区下面 的少量负电荷区是由之前的几次上行正地闪和一次 负地闪所残留的负电荷以及在云滴和冰雨之间感应 电荷的分离共同影响的. 但它们的主正和主负电荷 区中有大量的异极性电荷, 这些异极性电荷主要是 由前一次云闪过程植入, 虽然此时空中的电荷并没 有被立即消耗, 但其植入的异极性电荷会将原来存 在于主正与主负电荷区之间的强电场立即摧毁, 以 达到放电并降低空中强电场的作用(谭涌波等, 2007; Tao等, 2009). 与此同时, 由于底部次正电荷区并没 有被云闪所遍及, 其仍然保留足够的电荷量. 前一次 云闪过程的作用相当于降低了原来高空中的强电场, 而底部电场相对增强, 触发了上行地闪. 需要指出的 是, 虽然 sp3的底部次负电荷区对上行正地闪电产生 有抑制作用, 但是由于其主导作用的次正电荷区高 度很低, 次负电荷区的抑制作用对其影响不大, 上行 正地闪依然被触发。

剩下的 4 个自行触发的上行地闪中包括一个特殊 的上行正极性地闪 $\mathrm{sp} 2$, 其背景为反偶极性的电荷结 构(图1c), 即上部为主负电荷区, 下部为主正电荷区. 主正电荷区左边的长条负电荷是前一次上行正地闪 电所留下的感应电荷. 此次上行地闪发生在雷暴过 程的消亡阶段(表1), 其底部是主正电荷区, 其电荷 量相对较多并且高度也较低, 因此生成了足够强的 正位势阱，形成上行正地闪. 其余的 3 个自行触发的 上行地闪均为图 2 中的上行负地闪, Saba等(2008)观测 到的 $73 \%$ 的上行地闪都是从尖端触发的上行正先导 到达云内的负极性上行地闪. 图 $2 \mathrm{a}$ 为典型的正偶极 电荷结构, 此次上行负地闪发生在雷暴过程中的消 亡阶段, 此时很少发生云内闪电, 雷暴中的电场缓慢 增加, 并且消亡阶段的主负电荷区的垂直结构随着 降雨粒子的下降过程被拉长, 形成一段狭长的垂直 分布的主负电荷堆, 直至 $1 \mathrm{~km}$ 高度处, 这使得地面电 场被加强, 触发了上行负地闪. Jiang等(2014a)也观测 到自行触发的负极性上行地闪产生于雷暴活动的消 亡阶段, 此时伴随着降雨, 雷暴云中部的负电荷会下 降到近地面, 此时地面受负电场影响, 产生负极性上 行地闪. 而sn2、sn3两次上行正地闪较为特殊, 虽然 它们的电荷结构都可以看做三极性电荷结构, 不过 其底部电场极性以及位势阱均由主负电荷区起主导
作用, 次正电荷区的存在要么偏移 (图 $2 \mathrm{c}$ ), 要么量级 较小(图2e), 不足以对地面负位势阱形成干扰, 因此 仍然触发了上行负地闪. 与此同时, 通过表 1 不难发 现, sn2 以及 $\mathrm{sn} 3$ 均发生在雷暴过程的消亡阶段, 并为 山地雷暴个例, 主负电荷区离地面仅 $3 \mathrm{~km}$ 左右, 而电 荷堆高度的降低, 为上行地闪的自行触发提供了便 利条件. 许多观测发现负极性上行地闪大多出现在 冬季(Takagi等, 2006; Wang等, 2008; Lu等, 2009; Manhardt等, 2012), 并且指出冬季温度较低, 零度层 相比夏季会更加靠近地面, 使得负电荷区更靠近地 面, 可能是形成负极性的上行地闪的主要因素. 虽然 本文给出的是山地雷暴, 其零度层离地面的高度同 样较低, 其更容易产生自行触发的上行负地闪也与 观测结果具有一定的相似性.

由此可见, 其他闪电活动带来的迅速电场变化 可触发上行地闪, 与此同时, 由于雷暴云消亡阶段电 荷结构的下沉以及垂直结构被拉长, 以及冬季或山 地雷暴, 零度层更靠近地面等因素, 都是产生自行触 发的上行地闪的有利条件, 而其中上行正地闪多为 三极结构并在特殊情况下也可能会在反偶极结构下 触发, 而上行负地闪可在更为常见的偶极结构下起 始. 而已有的一些观测结果也证明了本文得到的上 行地闪的合理性 (Wang等, 2008; Warner等, 2012c; Diendorfer等, 2009; Wang等, 2012).

\subsection{2 上行先导传播范围以及分形情况探讨}

图1和图2中的上行地闪的电荷和电位的不同决 定了上行先导传播的水平、垂直范围也存在很大的差 异, 表3 给出了6次上行地闪的水平和垂直传播距离.

上行正地闪的通道水平距离从 1.0 到 $3.2 \mathrm{~km}$ 不等, 垂直距离从 3.1 到 $4.8 \mathrm{~km}$ 不等, 而上行负地闪的通道水 平距离从 4.0 到 $8.1 \mathrm{~km}$ 不等, 垂直距离从 5.0 到 $9.2 \mathrm{~km}$ 不 等. 相对于上行负地闪, 上行正地闪延伸范围更小. 另外, 上行正地闪 (sp1和 sp3) 的起始主要靠底部小的

表 36 次闪电先导的传播的水平与垂直距离

\begin{tabular}{ccc}
\hline 名称 & 水平距离 $(\mathrm{km})$ & 垂直距离 $(\mathrm{km})$ \\
\hline $\mathrm{sp} 1$ & 1.0 & 4.8 \\
$\mathrm{sp} 2$ & 1.3 & 4.6 \\
$\mathrm{sp} 3$ & 3.2 & 3.1 \\
$\mathrm{~s} 1$ & 8.1 & 9.2 \\
$\mathrm{sn} 2$ & 5.5 & 5.0 \\
$\mathrm{sn} 3$ & 4.0 & 5.5 \\
\hline
\end{tabular}


次正电荷区, 并且由于前次云内闪电的发生削弱了 上部电荷区，底部才会相对产生强电场，而底部次正 电荷区电荷量偏小, 生成的位势阱最大值偏小(表2), 导致上行地闪发展不够旺盛, 先导水平传播距离较 短; 而上行负地闪(sn1和 $\mathrm{sn} 2$ )多发生在消亡阶段，消 亡阶段虽然云体消散，不过起主导作用的是主负电 荷区, 雷暴云的底部为比较强大的负位势阱, 因此触 发的上行正先导会在负电荷区中充分的延伸, 形成 大量分支，而 sn3也是发生于雷暴云消亡阶段附近， 底部次正电荷区的存在并不足以改变底部负位势阱 的存在，因此sn3的先导在垂直经过底部次正电荷区 之后，几乎遍及主负电荷区，并形成强烈的水平扩 展. 对比表 2 及表 3 , 不难发现, 诱导触发的上行地闪 (sp1、sp3) 所对应的空间位势阱均较弱, 而闪电的延 伸范围也有限; 而所有的自行触发的上行地闪(sp2、 $\mathrm{sn} 1 、 \mathrm{sn} 2 、 \mathrm{sn} 3$ ) 空中均存在一个较强的位势阱, 而闪 电传播较为充分.

除此之外, 图3还给出了 6次上行地闪的分形特 征统计(Neimeyer等, 1984; Sanudo等, 1995; 谭涌波等, 2006; Tao等, 2009; Kawasaki等,2000), 定量给出上行 地闪通道的分叉情况对比. 其中 $r$ 为距上行地闪起始
点的格点数, $N(r)$ 为以 $r$ 为半径的圆内的通道格点数, $D$ 为拟合的分形维数. 从图中可以看出 $N(r)$ 和 $r$ 之间在 一定范围内有很好的幂指数关系 $N(r)=a * r^{D}$. 显然上 行正地闪分形维数 $D$ 较低, 平均在 1.20 左右, 并且有 的会呈现良好的线性分布，而上行负地闪的分形维 数 $D$ 很高, 平均在 1.60 以上, 其先导的分支明显多于 上行正地闪. 这是因为模拟得到的上行正地闪多为 诱导触发的上行地闪, 虽然云闪结束后可以触发上 行正地闪, 但云闪发生后会消耗大量的云内电荷, 使 电场整体降低, 很难为上行正地闪的充分延伸提供 条件, 除此之外, 次正电荷区对于上行正地闪的发生 起着主导作用，然而其电荷量相对较小，其触发的上 行正地闪电在起始阶段分叉很少, 呈现单支发展, 在 入云之后才有少量分支, 整体发展不旺盛; 而上行负 地闪多发生于消亡阶段的正偶极性电荷结构，此时 云底高度较低, 上行正先导很快就能入云, 并且主负 电荷区相对较大, 容易形成大量分支, 并且此时云内 闪电发生较少, 产生的上行负地闪多为自行触发的 上行地闪, 此时周围环境电场较强, 主负电荷区的电 荷量也积累的足够多, 因此上行负地闪相较于上行 正地闪延伸更充分、分支更多. 而图1c中的sp2比较特
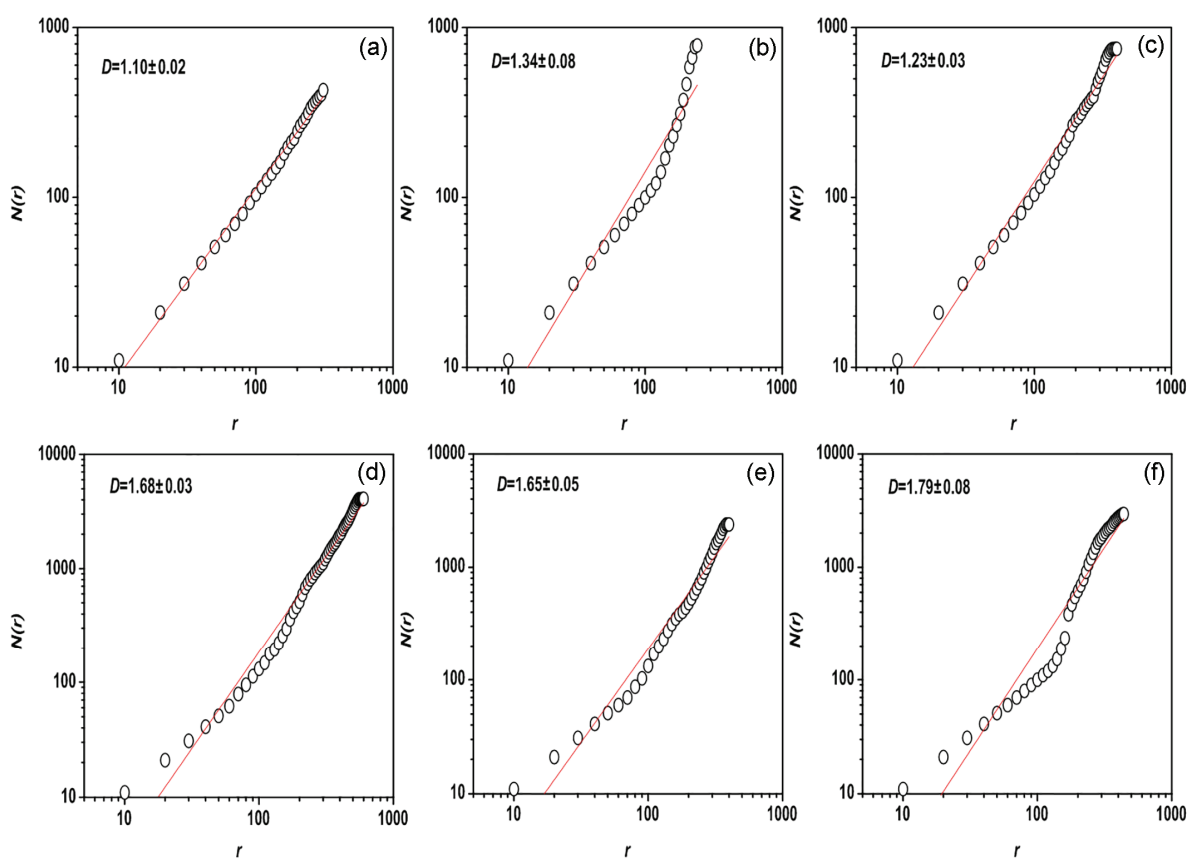

图 3 放电半径与通道格点数的幂指数关系

(a)为图 1a 的情况, (b)为图 1c 的情况, (c) 为图 1e 的情况, (d)为图 2a 的情况, (e)为图 2c 的情况, (f) 为图 2e 的情况. $r$ 为距闪电起始点的格点数, $N(r)$ 为以 $r$ 为半径的圆内的通道格点数, $D$ 为拟合的分形维数 
别, 它是发生在消亡阶段的上行正地闪, 并且其电荷 结构为反偶极性, 底部为正电荷区, 其电荷量比一般 的次正电荷区要大, 且高度更低, 空中位势阱也更 强, 因此 $\mathrm{sp} 2$ 的分形维数 $D$ 略大于其他的上行正地闪, 而却小于其他上行负地闪的分形维数.

\section{2 上行地闪触发类型的时间分布}

上行地闪分为两种触发类型: 一种是由其他闪 电活动引起的迅速电场变化而触发上行地闪为诱导 触发的上行地闪; 另一种是不依靠其他闪电活动, 而 靠静电荷的积累而产生强的静电场而触发的上行地 闪称为自行触发的上行地闪(Wang等, 2008). 两种触 发类型的上行地闪在雷暴发展阶段的触发概率有所 区别. 表4列出了诱导触发与自行触发的上行地闪分 别在雷暴发展的各个时期发生的概率. 这里的概率 指的是诱导触发以及自行触发的上行地闪在各个雷 暴云阶段的频次与其总频次之比.

表4中可以看出本文模拟的诱导触发的上行地闪 均发生在雷暴发展的成熟期与消散期, 并且在成熟 期的发生概率较高, 概率为 $89 \%$, 而在消散期发生概 率较低, 概率为 $11 \%$; 而自行触发的上行地闪也只发 生在雷暴发展的成熟期与消散期, 其在成熟期发生 的概率反而较低, 概率为 $22 \%$, 而在消散期发生的概 率较高, 概率为 $78 \%$. 很显然, 两种触发类型的上行 地闪均没有发生在雷暴的起始阶段, 此时雷暴发展 较弱, 地面电场达不到上行地闪的起始条件. 而诱导 触发的上行地闪之所以在成熟期发生概率较高, 可 能与雷暴的成熟期云内闪电活动较为频繁密切相关; 而自行触发的上行地闪在雷暴消散期发生的概率较 高, 主要与此时电荷结构的高度随着下沉气流而降 低、于此同时加大了地面环境电场强度息息相关. Warner等(2012c) 统计了 2004 2010 年 Rapid City 的 81 次上行地闪, 发现其中有 80 次上行地闪是由周 围的闪电活动触发的, 因为这些上行地闪多发生在 强对流雷暴天气中, 此时云内闪电活动也相应的强 烈; 而Zhou等(2012b)观测 2005 2009 年 Gaisberg Tower 产生的上行地闪, 发现 $87 \%$ 的上行地闪是自 触发上行闪电, 并且多发生冬季在非强对流雷暴中, 此时整体的雷暴高度偏低. 由此可见, 诱导触发的上 行地闪多发生在云内闪电活动频繁的雷暴成熟阶段, 而自行触发的上行地闪多发生在雷暴高度偏低的消 散阶段.
表 4 上行地闪触发类型的时间分布

\begin{tabular}{ccc}
\hline 雷暴的发展阶段 & 诱导触发概率 & 自行触发概率 \\
\hline 成熟阶段 & $89 \%$ & $22 \%$ \\
消散阶段 & $11 \%$ & $78 \%$ \\
\hline
\end{tabular}

\section{3 下行地闪与上行地闪对比}

自然界中存在着两种地闪, 一种是从云中触发 向地面发展的下行地闪, 另一种就是从地面触发向 云中发展的上行地闪. 同是地闪, 其触发条件是否相 同是值得深思的一个问题. 图 4 为模拟得到的 3 次不 同电荷结构下产生的下行地闪, 其中包括2次正地闪, 分别是PCG1(图4a、4b) 以及PCG2(图4c、4d), 和1次 负地闪 $\mathrm{NCG}$ (图4e、4f).

其中PCG2 是一次经典的始发于偶极电荷结构下 的下行正地闪, 而PCG1虽然是空间呈现三极电荷结 构, 但由于底部次正电荷区并不位于闪电起始位置 的正下方, 对下行的正先导没有阻碍作用, 因此, 其 正先导可以延伸至地面从而形成一次下行地闪过程. 通过触发下行正地闪时的空间电位图(图4b、4d)不难 发现, 空中以正位势占主导地位, 表明上部主正电荷 区量级大于中部主负电荷区, 闪电起始位置的电位 远远大于 $0 \mathrm{MV}$, 因此形成了下行正地闪(Tan 等, 2014a). 而图1给出的三次上行地闪均与离地面较近 的正电荷区有极大关系, 要么是三极结构下的次正 电荷区, 由于前次云闪过程摧毁了主正电荷区与主 负电荷区之间的强电场, 从而使得地面电场相对增 强, 产生了诱导触发的上行正地闪( $\mathrm{sp} 1$ 以及 $\mathrm{sp} 2$ ); 要 么是在反偶极电荷结构下, 随着底部正电荷区的下 沉以及增强, 从而产生了自行触发的上行正地闪. 可 见下行正地闪需要上部有一较强的正电荷区, 而上 行正地闪却恰恰相反, 需要的是离地面最近的电荷 区极性为正而且相对量级要足够大. 而从空间电位 分布也不难发现, 触发下行正地闪时, 空中的强正位 势阱位于高空, 而上行正地闪触发时的正位势阱位 于雷暴云的底部.

图 $4 \mathrm{e} 、 4 \mathrm{f}$ 为一次三极电荷结构下的下行负地闪, 底部的次正电荷区是产生下行负地闪的关键因素 (Clarence等, 1957; Wiliams, 1989; Pawar等, 2004; Qie 等, 2005; Nag等, 2009), 而其大小也对闪电类型有极 大的影响, 如果太小则云内闪电不会在下部产生, 如 果太大则容易生成反极性云闪, 只是处于一定范围 内才容易产生下行负地闪(谭涌波等, 2014). 而模拟 得到的上行负地闪则主要产生于偶极结构, 当雷暴 

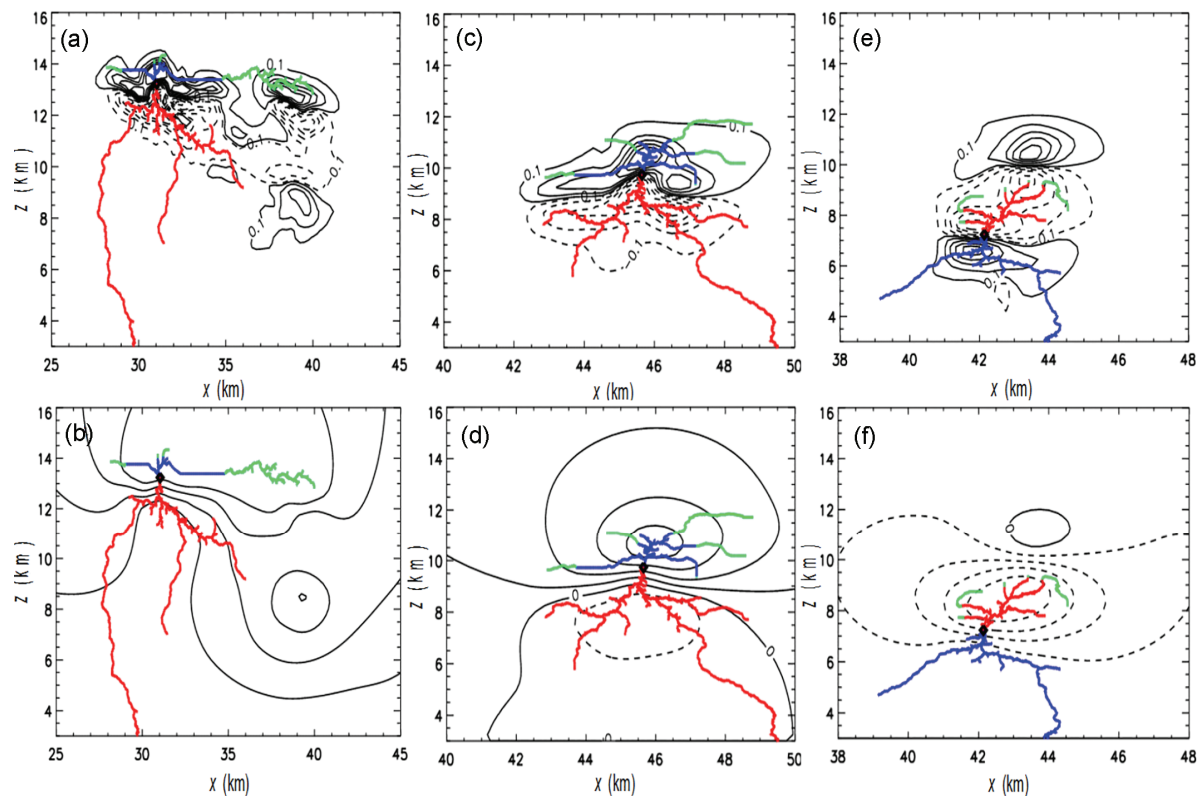

图 4 不同电荷结构下产生的下行正、负闪电通道与空间电位以及空间电荷的分布图

图中蓝色实线表示负先导通道，红色实线表示正先导通道，绿色表示后继回击通道; (a)、(c)、(e)中实线和虚线分别代表正、负电荷密度等值 线, 图中标定了 $\pm 0.1 \mathrm{nC} / \mathrm{m}^{3}$ 电荷密度等值线,等值线间隔为 $0.5 \mathrm{nC} / \mathrm{m}^{3}$; (b)、(d)、(f)中实线和虚线分别代表正、负电位等值线, 图中标定 0 等位 线, 正、负等位线间隔均为 $40 \mathrm{MV}$, 需要说明的是底边界也为 0 位势

云处于消亡阶段，电荷结构下沉或主负电荷区的垂 直结构随着降水粒子扩展, 才是产生自行触发的上 行负地闪的根本原因. 虽然 sn3也是始发于三极结构 下的一次上行负地闪，但不同于下行负地闪的是，其 底部次正电荷的存在主要是起阻碍作用, 其量级要 足够小, 只有当其不足以改变底部强负位势阱的分 布时，上行负地闪才有可能产生.

由此可见，上行地闪的触发条件与下行地闪截 然不同:下行正闪主要触发于偶极结构, 且上部的主 正电荷区占主导地位, 而上行正地闪要么是三极结 构下的诱导触发的上行地闪, 或在特殊的反偶极结 构下可能产生一次自行触发的上行地闪, 且下部正 电荷堆要足够大; 下行负地闪则始发于三极结构下 的中部主负电荷区与下部次正电荷区之间, 下部次 正电荷区的量级是下行负地闪始发的先决条件, 而 上行负地闪则主要起始于雷暴云消亡阶段的偶极结 构, 主负电荷区离地面较近且量级较大, 底部次正电 荷区对于上行负地闪而言起阻碍作用.

\section{4 讨论与结论}

目前对于上行地闪的研究主要还是通过实地观
测, 由于受上行地闪触发的随机性以及观测手段的 限制, 目前要获取上行地闪始发、传播时空中、地面 全部电参数难度较大, 而模式研究工作也比较少见, 并由于分辨率的限制, 大部分都是探讨下行地闪连 接过程中的上行先导过程. 基于此, 本文在已有的双 向先导随机模型（Mansell等, 2002; 谭涌波等, 2006) 的基础上，创建能够自行触发的上行地闪随机放电 参数化方案, 并耦合到雷暴云起、放电模式中, 再通 过大量敏感性实验, 模拟上行地闪的触发以及传播 入云过程, 探讨不同雷暴云电荷结构以及其他闪电 活动对上行地闪触发以及传播的有利条件, 比较产 生上行地闪与下行地闪的云中背景条件的差异. 主 要得到以下结论:

(1) 通过与大量国内外上行地闪观测结果的对 比，发现不管是闪电的空间形态、还是触发的电环境 特征等, 模拟得到的上行地闪都与观测结果具有较 好的一致性.

（2）其他闪电活动带来的快速电场变化可触发 上行地闪，与此同时，由于雷暴云消亡阶段电荷结构 的下沉以及垂直结构被拉长, 以及冬季或山地雷暴, 零度层更靠近地面等因素, 都是产生自行触发的上 
行地闪的有利条件. 而其中模拟得到的上行正地闪 多为诱导触发的上行地闪, 通常是三极电荷结构下 次正电荷区与地面之间的一种放电现象, 前次云闪 过程对空间环境电场的影响为其起始提供了有利条 件. 除此之外, 自行触发的上行正地闪也有可能发生 于特殊的反偶极电荷结构中; 而上行负地闪多发生 于偶极电荷结构中, 降水粒子的下沉以及冬季或山 地雷暴的零度层更靠近地面等因素是其发生的根本 原因. 除此之外, 三极性电荷结构也可以产生自行触 发的上行负地闪, 不过其主导作用的仍然是主负电 荷区, 底部次正电荷区对上行负地闪的触发反而起 了阻碍作用. 总体来说, 从电位分布上来看, 各种类 型的上行地闪触发时, 雷暴云底部都会有一个与其 极性相同的强位势阱.

(3) 上行地闪的闪电通道一开始呈单支垂直发 展趋势, 一般入云之后才会出现分支. 相对于上行负 地闪而言, 上行正地闪延伸范围有限, 上行正地闪发 展较弱, 分支较少; 诱导触发的上行地闪多发生于雷 暴的成熟期, 而自行触发的上行地闪多发生于雷暴 的消散期.

（4）上行地闪的触发条件与下行地闪截然不同: 下行正闪主要触发于偶极结构, 且上部的主正电荷 区占主导地位, 而上行正地闪则是需要下部的正电 荷堆要足够大, 要么是三极结构下的诱导触发的上 行地闪, 或在特殊的反偶极结构下可能产生一次自 行触发的上行地闪; 下行负地闪则始发于三极结构 下的中部主负电荷区与下部次正电荷区之间, 下部 次正电荷区的量级是下行负地闪始发的先决条件, 而上行负地闪则主要起始于雷暴云消亡阶段的偶极 结构, 主负电荷区离地面较近且量级较大是上行负 地闪触发的主要因素, 而底部次正电荷区的存在对 于上行负地闪起始而言起了阻碍作用.

本文在已有的双向先导随机模型基础上, 创建 能够自行触发的上行地闪随机放电参数化方案, 并 耦合到云模式中, 再通过大量敏感性实验, 模拟上行 地闪的触发以及传播入云过程, 分析了上行地闪触 发、传播的有利条件以及与下行地闪的云中背景条件 的差异. 国内外大量观测结果也证明了本模式的合 理性. 然而对于下行地闪诱导触发产生的上行地闪 而言(Warner等, 2012b; Zhou等, 2012b), 本文暂时还 无相应的模式设计, 因为观测结果表明其他闪电活
动会产生一个瞬时强电场, 在此之后几毫秒到几百 毫秒内会触发上行地闪, 而本模式的闪电没有时间 概念(谭涌波等, 2006), 为准静态下的闪电放电模拟, 暂时不考虑放电过程中辐射场对环境电场的影响. 除此之外, 关于正负先导阈值的差异性问题以及地 面电晕层对于地面环境电场的影响等问题, 还需更 深入的研究和探讨, 也是将来模式进一步构建和完 善的重点.

致谢感谢中国科学院大气物理研究所提供云模式.

\section{参考文献}

郭秀峰, 谭涌波, 朱俊儒, 梁忠武. 2012. 分辨率对建筑物周围大 气电场计算结果不确定性的影响. 中国气象学. S13 第十届防 雷减灾论坛——雷电灾害与风险评估.中国气象学会. 1: 11

郭秀峰, 谭涌波, 郭风霞, 师正, 王宁宁. 2013. 建筑物尖端对大 气电场畸变影响的数值计算. 应用气象学报, 2: 189-196

贺恒金金, 何俊佳, 钱冠军, 张丹丹, 黄萍. 2008. 棒-板长间隙正极 性流注生长概率模型及应用. 高电压技术, 34: 2047-2053

郄秀书, 张其林, 袁铁, 张廷龙. 2013. 雷电物理学 (地学卷). 北 京: 科学出版社. 291

任晓毓, 张义军, 吕伟涛, 陶善昌, 德庆措姆. 2010. 雷击建筑物 的先导连接过程模拟. 应用气象学, 21:450-457

谭涌波, 陶善昌, 祝宝友, 马明, 吕伟涛. 2006. 雷暴云内闪电双 层、分枝结构的数值模拟. 中国科学: 地球科学, 36: 486-496

谭涌波, 陶善昌, 祝宝友, 马明, 吕伟涛. 2007. 云闪放电对云内 电荷和电位分布影响的数值模拟. 地球物理学报, 50 : 1053-1065

谭涌波, 师正, 王宁宁, 郭秀峰. 2012. 随机性与电环境特征对地 闪击地点影响的数值模拟.地球物理学报, 55: 3534-3541

谭涌波, 梁忠武, 师正, 朱俊儒, 郭秀峰. 2014. 雷暴云底部正电 荷区对闪电类型影响的数值模拟. 中国科学: 地球科学, 44 : 2743-2752

Alessandro F D , Gumley J R. 2001. A "Collection Volume Method" for the placement of air terminals for the protection of structures against lightning. J Electrost, 50: 279-302

Becerra M, Vernon Cooray. 2006a. A self-consistent upward leader propagation model. J Phys D-Appl Phys, 39: 3708-3715

Becerra M, Vernon Cooray. 2006b. A simplified physical model to determine the lightning upward connecting leader inception. IEEE T Power Deliver, 21: 897-908

Berger K. 1967. Novel observations on lightning discharges, results and research on Mount San Salvatore. J Franklin Inst, 283: $478-525$

Berger K, Vogelsanger E. 1969. New results of lightning observations, in Planetary Electrodynamics. Proceedings of the 
4th International Conference on the Universal Aspects of Atmospheric Electricity, Tokyo. 1: 489-510

Carey L D, Murphy M J, McCormick T L, Demetriades N W S. 2005. Lightning location relative to storm structure in a leading-line, trailing-stratiform mesoscale convective system. J Geophys Res, 110: D03105

Chang J S, Beuthe T G, Hu G G, Stamoulis G, Janischewskyj W. 1985. Thundercloud electric field measurements in the $553 \mathrm{~m} \mathrm{CN}$ tower during 1978-1983. J Geophys Res, 94: 197-205

Clarence N D, Malan D J. 1957. Preliminary discharge processes in lightning flashes to ground. Q J R Meteorol Soc, 83: 161-172

Dienddorfer G, Pichler H, Mair M. 2009. Some parameters of negative upward-initiated lightning to the Gaisberg Tower (2000-2007). IEEE Trans Electromagn Aompat, 51: 443-452

Dienddorfer G, Zhou H L, Pichler H, Thottappillil R. 2011. Review of upward positive and bipolar lightning flashes at the Gaisberg Tower. 7th Asia-Pacific International Conference on Lightning, Chengdu. 263-267

Eriksson A J. 1978. Lightning and tall structures. SAIEE, 69: 238-253

Flache D, Rakov V A, Heidler F, Zischank W, Thottappillil R. 2008. Initial-stage pulses in upward lightning: Leader/return stroke versus M-component mode of charge transfer to ground. Geophys Res Lett, 35: L13812

Guimarães M, Araujo L, Pereira C, Mesquita C, Visacro S. 2014. Assessing currents of upward lightning measured in tropical regions. Atmos Res, 149: 324-332

Heldson J H, Wu G, Farley R D. 1992. An intracloud lightning parameterization scheme for a storm electrification model. J Geophys Res, 97: 5865-5884

Jiang R B, Sun Z L, Wu Z J. 2014a. Concurrent upward lightning flashes from two towers. Atmos Oceanic Sci Lett, 7: 260-264

Jiang R B, Qie X S, Wu Z J, Wang D F, Liu M Y, Lu G P, Liu D X. 2014b. Characteristics of upward lightning from a 325-m-tall meteorology tower. Atmos Res, 149: 111-119

Kawasaki Z, Matsuura K. 2000. Does a lightning channel show a fractal? Appl Energy, 67:147-158

Liu D X, Qie X S, Pan L X, Peng L. 2013. Some characteristics of lightning activity and radiation source distribution in a squall line over North China. Atmos Res, 132-133: 423-433

Lu W T, Wang D H, Zhang Y, Takagi N. 2009. Two associated upward lightning flashes that produced opposite polarity electric field changes. Geophys Res Lett, 36: L05801

Lu W T, Chen L W, Zhang Y, Ma Y, Gao Y, Yin Q Y, Chen S D, Huang Z H, Zhang Y J. 2012. Characteristics of unconnected upward leaders initiated from tall structures observed in Guangzhou. J Geophys Res, 117: D19211

MacGorman D R, Straka J M, Ziegler C L. 2001. A lightning parameterization for numerical cloud models. J Appl Meteorol,
40: 459-478

Manhardt M, Heidler F, Stimper K. 2012. The electric field of negative upward lightning strikes at the Peissenberg Tower, Germany. 31th International Conference on Lightning Protection, Vienna, Austria

Mansell E R, Macgorrnan D R, Ziegler C L, Straka J M. 2002. Simulated three-dimensional branched lightning in a numerical thunderstorm model. J Geophys Res, 107: 1-12

Mazur V, Ruhnke L H. 2011. Physical processes during development of upward leaders from tall structures. J Electrost, 69: 97-110

McEachron K B. 1939. Lightning to the Empire State Building. J Franklin Inst, 227: 149-217

Miki M, Rakov V A, Shindo T, Diendorfer G, Mair M, Heidler F, Zischank W, Uman M A, Thottappillil R, Wang D H. 2005. Initial stage in lightning initiated from tall objects and in rocket-triggered lightning. J Geophys Res, 110: D02109

Nag A, Rakov V A. 2009. Some inferences on the role of lower positive charge region in facilitating different types of lightning. Geophys Res Lett, 36: L05815

Niemeyer L, Pietronero L, Wiesman H L. 1984. Fractal dimension of dielectric breakdown. Phys Rev lett, 52: 1033-1036

Orville R E, Berger K. 1973. An unusual lightning flash initiated by an upward-propagating leader. J Geophys Res, 78: 4520-4525

Pawar S D, Kamra A K. 2004. Evolution of lightning and the possible initiation/triggering of lightning discharges by the lower positive charge center in an isolated thundercloud in the tropics. J Geophys Res, 109: D02205

Petrov N I, Waters R T. 1995. Determination of the striking distance of lightning to earthed structures. Math Phys Sci, 450: 589-601

Pichler H, Diendorfer G, Mair M. 2010. Some parameters of correlated current and radiated field pulses from lightning to the Gaisberg Tower. IEEJ Trans Electron Electromagn Eng, 5: 8-13

Qie X S, Zhang T L, Chen C P, Zhang G S, Zhang T, Wu Z W. 2005. The lower positive charge center and its effect on lightning discharges on the Tibetan Plateau. Geophys Res Lett, 32: L05814

Qie X S, Soula, Chauzy S. 1994. Influence of ion attachment on vertical distribution of electric field and charge density under thunderstorm. Ann Geophys, 12: 1218-1228

Rakov V A, Uman M A. 2003. Lightning: Physics and Effects. Cambridge: Cambridge University Press. 687

Romero C, Paolone M, Rachidi F, Rubinstein M, Rubinstein A, Diendorfer G, Schulz W, Bernardi M, Nucci C A. 2011. Preliminary comparison of data from the Säntis Tower and the EUCLID lightning location system. 6th International Symposium on Lightning Protection, IEEE, Fortaleza, Brazil

Saba M M F, Cummins L, Warner T A, Krider E P, Campos L Z S, Ballarotti M G, Jr O P, Fleenor S A. 2008. Positive leader characteristics from high-speed video observations. Geophys Res Lett, 35: L07802 
Sanudo J, Gomez J B, Castano F, Pacheco A F. 1995. Fractal dimension of lightning discharge. Nonlinear Process Geophys, 2: 101-106

Shi Z, Tan Y B, Tang H Q, Sun J, Yang Y, Peng L, Guo X F. 2015. Aerosol effect on the land-ocean contrast in thunderstorm electrification and lightning frequency. Atmos Res, 164-165: $131-141$

Takagi N, Wang D H, and Watanabe T. 2006. A study of upward positive leaders based on simultaneous observation of E-fields and high-speed images. Trans Inst Electr Eng Jpn, 126: 256-259

Tan Y B, Tao S C, Zhu B Y. 2006. Fine-resolution of the channel structures and propagation features of intracloud lightning. Geoghys Res Lett, 33: L09809

Tan Y B, Tao S C, Liang Z W, Zhu B Y. 2014a. Numerical study on relationship between lightning types and distribution of space charge and electric potential. J Geophys Res, 119: 1003-1014

Tan Y B, Guo X F, Zhu J R, Shi Z, Zhang D D. 2014b. Influence on simulation accuracy of atmospheric electric field around a building by space resolution. Atmos Res, 138: 301-307

Tao S C, Tan Y B, Zhu B Y, Ma M, Lu W T. 2009. Fine-resolution simulation of cloud-to-ground lightning and thundercloud charge transfer. Atmos Res, 91: 360-370

Wang D H, Takagi H, T Watanabe, Sakurano H, Hashimoto M. 2008. Observed characteristics of upward leader that initiated from a windmill and its lightning protection tower. Geoghys Res Lett, 35: L02803

Wang D H , Takagi N. 2012. Characteristics of winter lightning that occurred on a windmill and its lightning protection tower in Japan. IEEJ Trans Power Energy, 132: 568-572
Warner T A. 2012. Observations of simultaneous upward lightning leaders from multiple tall structures. Atmos Res, 117: 45-54

Warner T A, Saba M M F, Orville R E. 2012a. Characteristics of upward leaders from tall towers. 22th International Lightning Detection Conference, Vaisala, Boulder, Colo

Warner T A, Saba M M F, Rudge S, Bunkers M, Lyons W A, Orville R E. 2012b. Lightning-triggered upward lightning from towers in Rapid City, South Dakota. 22th International Lightning Detection Conference, Vaisala, Boulder, Colo

Warner T A, Cummins K L, Orville R E. 2012c. Upward lightning observations from towers in Rapid City, South Dakota and comparison with National Lightning Detection Network data, 2004-2010. J Geophys Res, 117: D19109

Warner T A, Lang T J, Lyons W A. 2014. Synoptic scale outbreak of self-initiated upward lightning (SIUL) from tall structures during the central U.S. blizzard of 1-2 February 2011. J Geophys Res, 119: 9530-9548

Wiliams E R. 1989. The tripole structure of thunderstorms. J Geophys Res, 94: 13151-13167

Zhou H L, Diendorfer G, Thottappillil R, Pichler H, Mair M. 2012a. Characteristics of upward positive lightning flashes initiated from the Gaisberg Tower. J Geophys Res, 117: D06110

Zhou H L, Diendorfer G, Thottappillil R, Pichler H, Mair M. 2012 b. Measured current and close electric field changes associated with the initiation of upward lightning from a tall tower. J Geophys Res, 117: D08102

Ziegler C L, MacGorman D R, Dye J E, Ray P S. 1991. A model evaluation of noninductive graupel-ice charging in the early electrification of a mountain thunderstorm. J Geophys Res, 96: 12833-12855 